\title{
Inhibition of microRNA-874 ameliorates cardiomyocyte apoptosis and improves cardiac function in the peripartum cardiomyopathy of Gaq transgenic mice
}

\author{
Chenchen ZHOU", Jindan PEI", Xiaobo ZHAO\#, Shengyi GU, Yuelin WU, Sheng WAN, Ronghua CHE, Zhimin \\ HAN, Xiaolin HUA*
}

Department of Obstetrics, Shanghai First Maternity and Infant Hospital, Tongji University School of Medicine, Shanghai 201204, China.

${ }^{*}$ Correspondence: xiaolin_hua@tongji.edu.cn

https://doi.org/10.37175/stemedicine.v2i5.75

\begin{abstract}
It is widely reported that microRNAs (miRNAs, miRs) play critical roles in the occurrence and development of peripartum cardiomyopathy (PPCM). Here, we aimed to explore the biological role of miR- 874 and its underlying mechanisms in PPCM. To this purpose, a mouse model of PPCM was established through cardiacspecific overexpression of Gaq. Transthoracic echocardiography and left ventricular catheterization were used to examine the cardiac functions and hemodynamics. Apoptosis of the cardiomyocytes was determined by the terminal deoxynucleotidyl transferase dUTP nick end labeling assay. The direct interaction between miR-874 and signal transducer and activator of transcription 3 (STAT3) was confirmed by luciferase assay. The expression of apoptosis-related genes and proteins was evaluated by real-time quantitative reverse transcription polymerase chain reaction and Western blot, respectively. The results demonstrated that miR-874 inhibition significantly increased the survival and cardiac functions of pregnant Gaq transgenic mice. In addition, miR-874 inhibitor ameliorated cardiomyocyte apoptosis, downregulated both mRNA and protein levels of Bax, while upregulated those of Bcl-2. Further, STAT3 was found to be a direct target of miR-874. In addition, miR-874 inhibition increased the expression of STAT3 and janus kinase 2. In summary, miR-874 inhibition could improve cardiac functions and suppress cardiomyocyte apoptosis by targeting STAT3 during PPCM in Gaq transgenic mice.
\end{abstract}

Keywords: MicroRNA-874 · PPCM · Left ventricular function · Cardiomyocyte apoptosis · STAT3

\section{Introduction}

Peripartum cardiomyopathy (PPCM) is a type of cardiac disease of unknown etiology occurred in the third trimester of pregnancy or even months after delivery, without other causes of heart failure (1). The left ventricular ejection fraction of PPCM patients is less than $45 \%$ (similar to dilated cardiomyopathy) with or without left ventricular dilatation (2). The clinical manifestations of PPCM include cardiac enlargement (mainly left ventricular enlargement), heart failure (dyspnea, fatigue and peripheral edema) and

Received: Nov 26, 2020; Accepted: Dec 22, 2020.

(c) The Author(s). 2020 This is an Open Access article distributed under the terms of the Creative Commons License (http://creativecommons.org/licenses/by/4.0/) which permits unrestricted use, distribution, and reproduction in any medium or format, provided the original work is properly cited. thromboembolism (such as pulmonary and/or cerebral embolism) (3). Malignant arrhythmias or sudden cardiac death was occasionally seen (3). Although the pathogenesis of PPCM is not clear, there are several potential risk factors including inflammatory response, viral infection, autoimmune response, oxidative stress and prolactin, as well as some genetic factors (4). Currently, the effective treatments of PPCM adopt standard heart failure treatment and supportive treatment. However, there is no specific treatment method for PPCM (5). Herein, it is important to explore the molecular mechanisms underlying PPCM to develop corresponding new treatment schemes.

MicroRNAs (miRNAs, miRs), as small non-coding RNAs, are believed to participate in multiple biological processes, including cell proliferation, apoptosis, differentiation and growth (6). MiRNAs are reported to 
exert important functions in various cardiovascular diseases, such as myocardial hypertrophy, myocardial infarction, heart failure, hypertension and arrhythmia (7). Most current studies on miR-874 have focused on its inhibitory role in cancer, but its role in cardiovascular diseases remains unclear. It was suggested that miR874 inhibitors could suppress myocardial cell necrosis by targeting cysteinyl aspartate specific proteinase (caspase)-8 (8). In addition, miR-874 inhibition could protect cardiomyocytes and inhibit cardiomyocyte apoptosis caused by ischemia-reperfusion by targeting signal transducer and activator of transcription 3 (STAT3) in mice (9). Besides, STAT3 is thought to participate in cardio-protection by inhibiting reactive oxygen-induced pregnancy oxidative stress (10). Studies have shown that female mice with a cardiomyocyte-specific loss of STAT3 exhibit the PPCM phenotypes $(11,12)$. Reduction of STAT3 activates cathepsin D and increases oxidative stress, which resulted in the inhibition of endothelial cell migration and proliferation, as well as cell apoptosis and impairment of heart functions by cleaving prolactin into 16k Da fragments (13). Gaq, a member of the Gq/11 subfamily of $\mathrm{G}$ protein, is a functional unit of the Gaq protein encoded by Gnaq (14). It is documented that mice with cardiac-specific overexpression of Gaq exhibit systolic dysfunction and baseline cardiac hypertrophy (15). The mice were susceptible to heart failure when challenged with hemodynamic overload (15). We therefore hypothesized that regulation of miR-874 expression should be able to modulate the myocardial function and myocardial apoptosis during PPCM.

\section{Materials and Methods \\ Animal model}

The Gaq transgenic mice were established as previously described (16). The oocytes were microinjected with 1,000 copies of the fragment and were subsequently implanted into pseudo-pregnant females. Genomic Southern analysis was used to screen the presence of the transgene in threeweek old mouse pups. Treatment with miR-874 inhibitor was conducted on the 12th day of pregnancy, and tissue samples were obtained 23 days later, on the 14th day postpartum. Pregnant Gaq transgenic mice (10 weeks old) were divided into four groups ( $\mathrm{n}=24$ each group): 1) agematched, non-pregnant female wild-type mice, 2) Gaq transgenic mice, 3) pregnant Gaq transgenic mice, and 4) Gaq transgenic mice treated with miR-874 inhibitor. Animal studies were reviewed and approved by the Ethics Commitment of Shanghai First Maternity and Infant Hospital.

\section{MiR-874 inhibition}

MiR-874 antagomir negative control (antagomirNC) and antagomir were provided by GenePharma (Shanghai, China). All the $3^{\prime}$-ends were conjugated to cholesterol, and the bases were $2^{\prime}-\mathrm{OMe}$ modified. The sequence of miR-874 antagomir was 5'-UCGGUCCCUCGGGCCAGGGCAG-3'. The chemically modified oligonucleotides (5'-CAGUACUUUUGUGUAGUACAA-3') was used as the negative control (miR-874-NC). Mice were injected intravenously with miR-874 antagomir or its negative control $(0.2 \mathrm{~mL})$ for three consecutive days at a dose of $30 \mathrm{mg} / \mathrm{kg}$ body weight per injection.

\section{Echocardiography and cardiac hemodynamics}

Echocardiography was applied to determine the left ventricular function by using Vevo 2100 (Visual Sonics, Canada). Echocardiographic examination was performed on the surviving mice, and the four groups of mice were mixed and numbered for anesthesia and examination by an assistant using a double-blind method. The mice to be tested were anesthetized by inhalation of isoflurane, and the heart rate was kept constant at $(400 \pm 20)$ times/ min during the examination. A heated plate was used to keep the body temperature of the mice constant during the examination. In order to ensure the imaging quality, mice were kept in a fixed position, with hair removed and skin prepared by applying an appropriate amount of ultrasonic couplant to the chest skin. The values obtained in the examination were averages of 5 consecutive cardiac cycles. A high-frequency probe was placed on the left thoracic side of the mouse, with the probe notch pointed toward the mouse's head, and rotated $30^{\circ}$ to $45^{\circ}$ counterclockwise to switch to M-mode. The sampling line was placed at the level of the mitral valve chordae, perpendicular to the ventricular septum and the posterior wall of the left ventricle. The scanning speed of the curve was $200 \mathrm{~mm} / \mathrm{s}$. The left ventricular fraction shortening (LVFS) and left ventricular end-diastolic dimension (LVEDD) were then obtained. Left ventricular catheterization was measured by a multichannel physiological recorder (Beijing B\&E Teksystems, Beijing, China). Mice were anesthetized with light methoxyflurane and were placed supine on a heating pad. Then a pressure micro catheter was used to retrograde into the left ventricle of the mouse, which was connected to the Power Lab workstation to obtain the left ventricular end-diastolic pressure (LVEDP), left ventricular systolic pressure (LVSP), $-\mathrm{dp} / \mathrm{dt}_{\max }$ and $+\mathrm{dp} / \mathrm{dt}_{\max }$.

\section{Cell apoptosis}

The left ventricular tissues of the experimental mice were derived and fixed in paraformaldehyde (4\%), dehydrated and embedded in paraffin. They were further cut into 3-4 $\mu \mathrm{m}$ slices, attached on a poly-lysine adhesion slide, and baked at $65^{\circ} \mathrm{C}$ for $1 \mathrm{~h}$. Cell apoptosis was further detected by One Step TUNEL Apoptosis Assay Kit (Beyotime, Shanghai, China) according to the manufactures' instructions.

\section{Reactive oxygen species (ROS) measurement}

The left ventricular tissues from indicated mice were freshly isolated and digested with trypsin for $15 \mathrm{~min}$, and gently blown for $1 \mathrm{~min}$ using a pipette. The isolated cells 
were then plated in 24 -well plates $\left(5 \times 10^{5}\right.$ cells/well $)$. Intracellular ROS levels were evaluated with DCFHDA. The cells were incubated with DCFH-DA $(10 \mu \mathrm{M})$ at $37^{\circ} \mathrm{C}$. Fluorescent signal intensity of DCF was examined at $488 \mathrm{~nm}$ (excitation wavelength) and $525 \mathrm{~nm}$ (emission wavelength).

\section{qRT-PCR}

The total RNA from isolated left ventricular tissues was extracted with Trizol reagent (Life Technologies, Carlsbad, CA). Then, the RNA was reverse transcribed into cDNA by a reverse transcription kit (Life Technologies). qRT-PCR was conducted using $2 \times$ SYBR Green PCR Master Mix (Life Technologies) to detect the levels of Bax, Bcl-2, miR874 , and STAT3. $\beta$-actin or U6 (for miR-874) was used as the internal control. The following primers were used: U6:

F: CTCGCTTCGGCAGCACA; R: AACGCTTCAGAATTTGCGT. STAT3:

F: GAAACAACCAGTCTGTGACCAG; R: CACGTACTCCATTGCTGACAAG. miRNA-874:

F: TGCGGCGGCCCCACGCACCAG; R: CCAGTGCAGGGTCCGAGGT.

Bax:

F: CTGAGCTGACCTTGGAGC; R: GACTCCAGCCACAAAGATG. Bcl-2:

F: TGCACCTGAGCGCCTTCAC; R: TAGCTGATTCGACCATTT.

Bcl-XL:

F: ACATCCCAGCTTCACATAACCC; R: CCATCCCGAAAGAGTTCATTCAC. JAK2:

F: GCAAAGGTAACTTCGGGAGTGT; R: AGTCTCGGAGGTGCTCTTCAG.

$\beta$-actin:

F: GACGGCCAGGTCATCACTATTG;

R: AGGAAGGCTGGAAAAGAGCC.

The relative gene expression was calculated using the $2^{-\Delta \Delta \mathrm{Ct}}$ relative quantitative method.

\section{Western blot}

Western bolt analysis was carried out following common protocol. The total protein of isolated left ventricular tissues was extracted. Sodium dodecyl sulfate lysate was added. $20 \mu \mathrm{L}$ protein was prepared, then loaded in $12 \%$ polyacrylamide gel electrophoresis. After transferred to the membranes, the membranes with the protein were blocked by Tris-buffered saline Tween-20 containing $5 \%$ bovine serum albumin for $1 \mathrm{~h}$ at room temperature. The primary antibodies including anti-Bcl-2 (ab59348, 1:1000), anti-Bax (ab32503, 1:1000), anti-STAT3 (ab68153, 1:1000), anti-janus kinase 2 (JAK2) (ab108596, 1:1000) were purchased from Abcam (Cambridge, MA). GAPDH (ab8245, 1:3000) was used as a loading control. The primary antibodies were incubated at $4{ }^{\circ} \mathrm{C}$ overnight.
Horseradish peroxidase-conjugated secondary antibodies (Invitrogen) were used for further incubation for $2 \mathrm{~h}$.

\section{Dual luciferase reporter gene assay}

The sequences of STAT3 3'-untranslated region (UTR) including the wild-type binding site of miR-874 or the mutated binding site were cloned into the pMIR vector (Promega Corporation, WI, USA). MiR-874 mimics was purchased from GenePharma (Shanghai, China). The relative luciferase activity was measured by Luciferase Dual Assay Kit (Thermo Fisher Scientific, Waltham, MA).

\section{Statistics}

The data were shown as mean \pm standard deviation (SD). Survival comparison between groups was performed by Kaplan-Meier analysis with a Log-rank (MantelCox) test. To analyze data between multiple groups, one-way ANOVA followed with a Tukey's post hoc test was performed. Statistically significant difference was accepted at $\mathrm{p}<0.05$.

\section{Results \\ MiR-874 inhibitor significantly decreases the mortality of the PPCM mice}

To evaluate the activity of miR-874 inhibitor on the mortality of PPCM in the Gaq mice, a Kaplan-Meier survival analysis was employed. The mice were randomly divided into four groups, with 24 mice in each group. Administration of miR-874 inhibitor on the 12th day after pregnancy was considered as the beginning of the experiment, and then the experiment was terminated after 23 consecutive days (on the 14th day after delivery). Study design of the research was shown in Figure S1. It was shown that the mortality in the pregnant Gaq transgenic mice was 10 out of 24 , whereas miR-874 antagonist decreased the mortality to 3 out of 24 in the pregnant Gaq transgenic mice (Figure 1). Of note, we found that control antagomir had little effect on the survival rate of the pregnant Gaq transgenic mice (Figure S2). Thus, treatment with miR-874 inhibitor significantly decreased the mortality of PPCM in the Gaq transgenic mice.

\section{MiR-874 inhibitor modulates the left ventricular function in PPCM}

To further investigate the role of miR-874 in PPCM, echocardiography was applied to determine the left ventricular function in the mice. At the 14th day after delivery, LVEDD and LVFS of the age-matched, female non-pregnant wild-type $(n=24)$ and Gaq transgenic mice $(n=24)$, pregnant Gaq transgenic mice $(n=14)$ and Gaq transgenic mice treated with miR-874 inhibitor $(n=21)$ were compared. Consistent with the previous literature, the LVEDD was higher in Gaq transgenic mice than in wild-type mice before pregnancy. In addition, the pregnant Gaq transgenic mice showed a further increased LVEDD, which could be reversed by treatment with miR- 


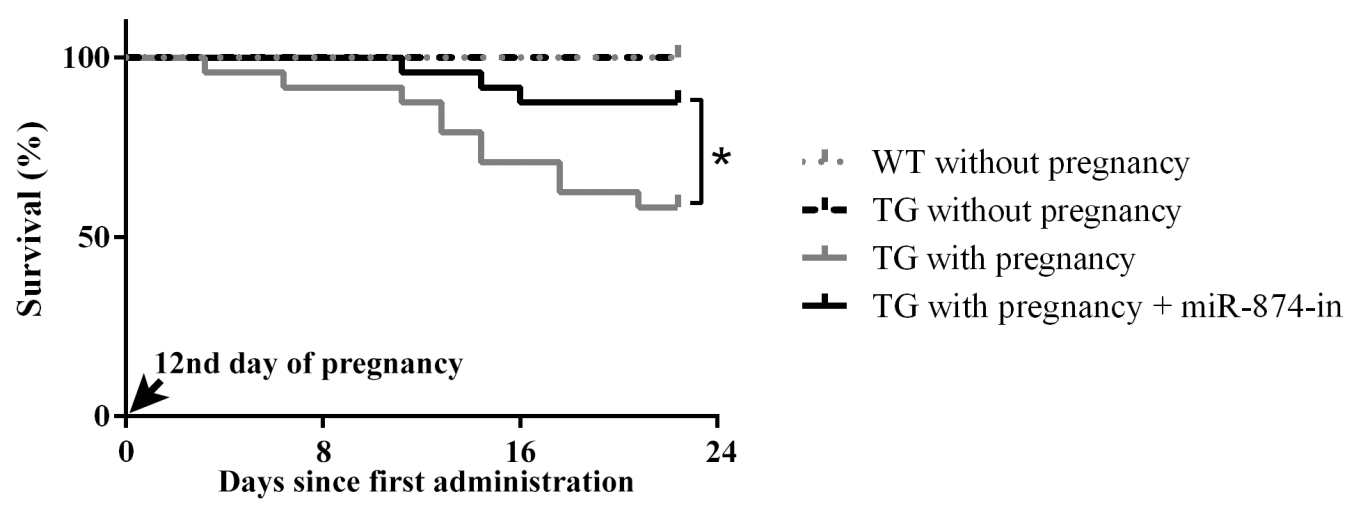

Figure 1. Kaplan-Meier survival analysis. All-cause mortality over course of experiment starting at day 12 after conception and 23 days continuous before euthanasia. All animals that entered study were included in this analysis. ${ }^{*} p<0.05$ between the indicated groups, Log-rank (Mantel-Cox) test.

874 inhibitor (Figure 2A). Next, we found that the LVFS was lower in Gaq transgenic mice than in wild-type mice before pregnancy. Besides, the pregnant Gaq transgenic mice showed a further decreased LVFS, whereas treatment with miR-874 inhibitor reversed this trend (Figure 2B). In addition, the control antagomir had no significant effect on the left ventricular functions in the pregnant Gaq transgenic mice (Figure S3). Therefore, our results demonstrated that treatment with miR-874 inhibitor could partially rescue the left ventricular dilation and contractile dysfunction.

\section{MiR-874 inhibitor modulates the left ventricular hemodynamics in PPCM}

The LVSP was decreased in Gaq transgenic mice compared with the wild-type mice before pregnancy, and the pregnant Gaq transgenic mice showed a further decreased LVSP. The treatment using miR-874 inhibitor significantly increased the LVSP to a level similar to the non-pregnant Gaq transgenic mice (Figure 3A). Besides, the LVEDP was obviously higher in Gaq transgenic mice than in wild-type mice before pregnancy, and further increased in the pregnant Gaq transgenic mice, which was remarkably decreased by miR-874 inhibitor (Figure 3B). In addition, we found that $+\mathrm{dP} / \mathrm{dt}$ and $-\mathrm{dP} / \mathrm{dt}$ was decreased in Gaq transgenic mice compared with the wild-type mice before pregnancy, and was even further decreased in the pregnant Gaq transgenic mice. The treatment using miR874 inhibitor rescued the reduced $+\mathrm{dP} / \mathrm{dt}$ and $-\mathrm{dP} / \mathrm{dt}$ during PPCM in the Gaq transgenic mice (Figure 3C and D).

\section{MiR-874 inhibitor significantly suppresses the myocardial apoptosis}

Since cardiomyocyte apoptosis is involved in perinatal cardiomyopathy, we examined the effect of miR-874 antagonist on cardiomyocyte apoptosis. By TUNEL staining assay, we found that the cell apoptosis was dramatically increased in Gaq transgenic mice compared with the wild-type mice before pregnancy, and the pregnant Gaq transgenic mice showed a further increase in the percentage of apoptotic cells, which was consistent with the previous literature. Notably, miR-874 inhibitor significantly decreased the percentage of apoptotic cells in the left ventricular tissues in the Gaq transgenic mice (Figure 4A and B). Therefore, we demonstrated that inhibition of miR-874 effectively suppressed the myocardial apoptosis.

\section{MiR-874 inhibitor regulates the expression of Bax and Bcl-2 in PPCM of the Gaq transgenic mice}

The expression of two apoptosis-related genes, Bax and $\mathrm{Bcl}-2$, were further determined in the left ventricular myocardial tissues to explore the potential of miR-874 on cell apoptosis. Both the mRNA and the protein levels of Bax were increased in Gaq transgenic mice compared with the wild-type mice before pregnancy, and the pregnant Gaq transgenic mice showed a further increased the Bax expression (Figure $\mathbf{5 A}, \mathbf{C}$, and $\mathbf{E}$ ). The treatment using miR-874 inhibitor significantly decreased the expression of Bax to a level similar as the non-pregnant Gaq transgenic mice (Figure 5A, C, and E). In addition, the mRNA and the protein levels of Bcl-2 were decreased in Gaq transgenic mice compared with the wild-type mice before pregnancy, and the pregnant Gaq transgenic mice showed a further decreased Bcl-2 expression (Figure 5B, D, and E). Importantly, miR-874 inhibitor significantly upregulated the expression of Bcl-2 (Figure $5 \mathrm{~B}, \mathrm{D}$, and $\mathrm{E}$ ). Various studies have shown that reactive oxygen species (ROS), such as oxygen free radicals and their derivatives, are closely related to cell apoptosis. Therefore, we examined the ROS production and BclXL expression in each group. These results indicated that the ROS production was increased in the Gaq transgenic mice compared with the wild-type mice before pregnancy, and the pregnant Gaq transgenic mice showed further increased ROS production, which was significantly reversed by miR-874 inhibitor (Figure 6A). In addition, the mRNA level of Bcl-XL was decreased in the Gaq transgenic mice compared with the wild-type mice before pregnancy, and the pregnant Gaq transgenic mice showed further decreased Bcl-XL expression (Figure 6B). Importantly, miR-874 inhibitor significantly upregulated 
A

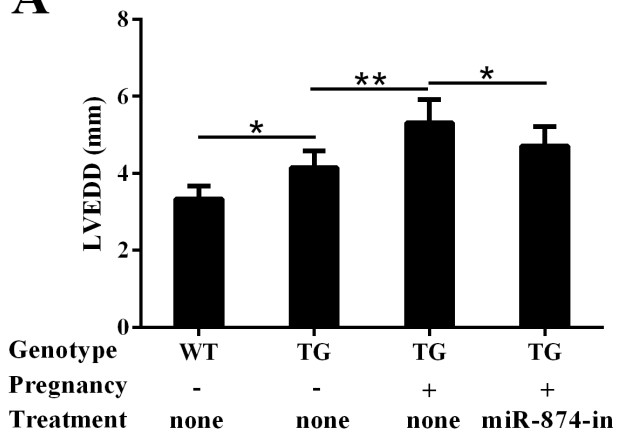

B

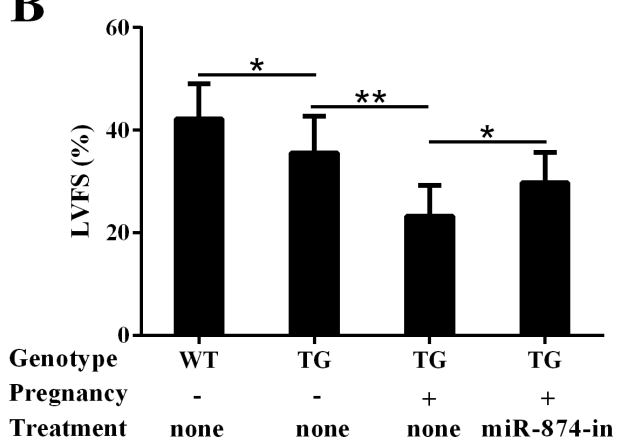

Figure 2. Echocardiographic analysis of left ventricular function. LVEDD (A) and LVFS (B) in survived mice from each group. Agematched, female non-pregnant wild-type (WT, n=24) and Gaq transgenic mice (GT, n=24), pregnant Gaq transgenic mice $(n=14)$ and Gaq transgenic mice treated with miR-874 antagonist (miR-874-in, $n=21$ ) were compared. Data are presented as mean $\pm S D$. ${ }^{*} p<0.05$, ${ }^{* *} p<0.01$ between the indicated groups. LVEDD: left ventricular end-diastolic dimension, LVFS: left ventricular fraction shortening.

the expression of Bcl-XL (Figure 6B).

\section{MiR-874 directly targets and suppresses the expression of STAT3}

Since miR-874 was critical in regulating cardiomyocyte apoptosis in PPCM of the Gaq transgenic mice, we examined miR-874 expression in all four groups. The expression of miR-874 was increased in Gaq transgenic mice compared with the wild-type mice before pregnancy, and the pregnant Gaq transgenic mice showed a further increase. MiR-874 inhibitor successfully downregulated the expression of miR-874 (Figure 7A). The mRNA levels of STAT3 and JAK2 were decreased in Gaq transgenic mice compared with wild-type mice before pregnancy, and the pregnant Gaq transgenic mice showed a further decrease in STAT3 and JAK2 expression (Figure 7B and C). Notably, miR-874 inhibitor significantly upregulated the expression of STAT3 (Figure 7B and C), suggesting a correlation between miR-874 and STAT3. Western blot analysis further confirmed that the patterns of STAT3 and JAK protein expression were consistent with their mRNA levels, respectively (Figure 7D-F). Next, to verify the direct interaction between miR-874 and STAT3, the predicted binding sequences of miR-874 with wild-type or mutant 3'-UTR region of STAT3 mRNA were subcloned
A

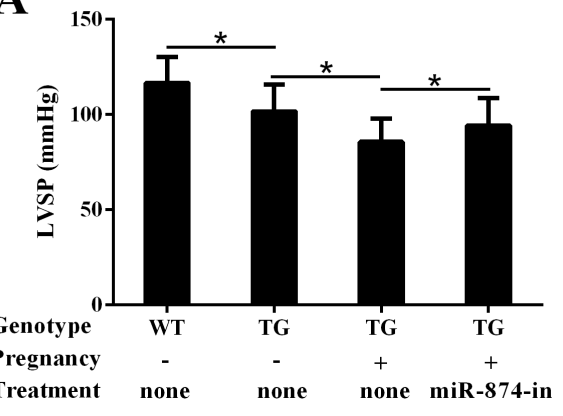

C

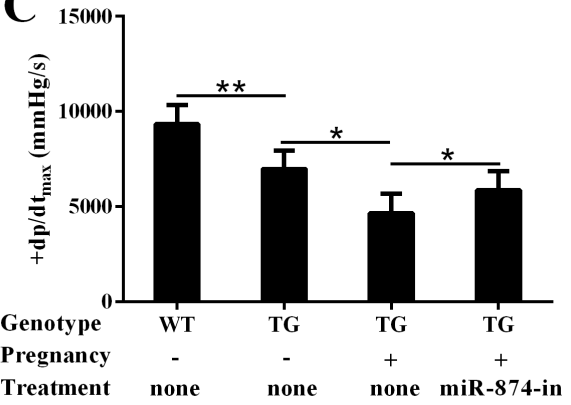

B
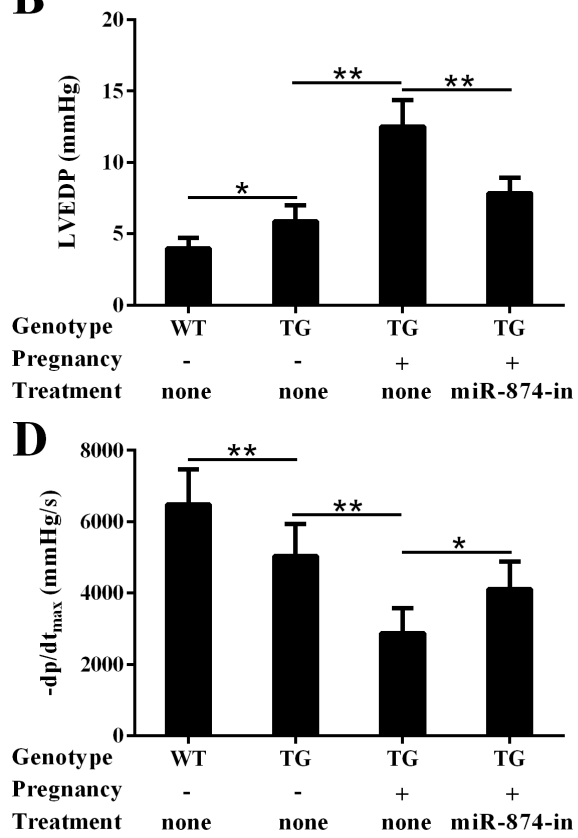

Figure 3. Analysis of left ventricular hemodynamics. LVSP (A), LVEDP (B), +dp/dtmax (C) and -dp/dtmax (D) in survived mice from each group. Age-matched, female non-pregnant wild-type (WT, $n=24)$ and Gaq transgenic mice (GT, $n=24)$, pregnant Gaq transgenic mice $(n=14)$ and Gaq transgenic mice treated with miR-874 antagonist (miR-874-in, $n=21$ ) were compared. Data are presented as mean $\pm S D$. ${ }^{*} p<0.05,{ }^{* *} p<0.01$ between the indicated groups. LVSP: left ventricular systolic pressure, LVEDP: left ventricular end-diastolic pressure. 
A
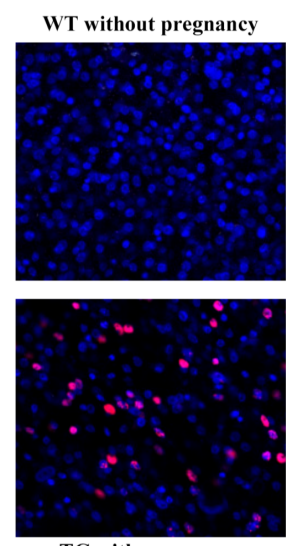

TG with pregnancy
B
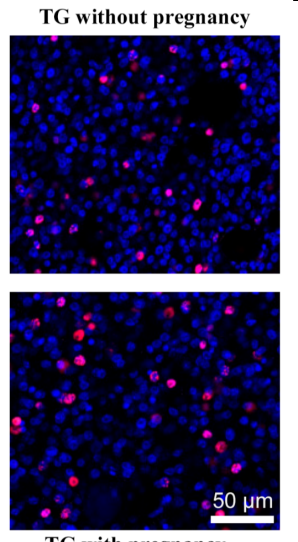

TG with pregnancy treated with miR-874-in

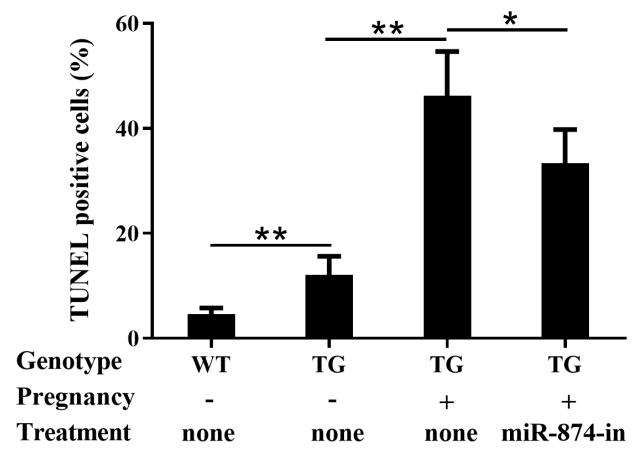

Treatment none none none miR-874-in

Figure 4. Inhibition of miR-874 ameliorated left ventricular cell apoptosis in PPCM of Gaq transgenic mice. (A) Representative TUNEL staining for detecting left ventricular cell apoptosis among different groups. Cells marked with red indicated apoptosis cells. (B) The percentage of apoptosis ratio among different groups from the TUNEL staining. Age-matched, female non-pregnant wild-type (WT, $\mathrm{n}=6$ ) and Gaq transgenic mice ( $\mathrm{GT}, \mathrm{n}=6)$, pregnant Gaq transgenic mice $(\mathrm{n}=6)$ and Gaq transgenic mice treated with miR-874 antagonist (miR-874-in, $n=6$ ) were compared. Data are presented as mean \pm SD. ${ }^{*} p<0.05,{ }^{* *} p<0.01$ between the indicated groups.

to a luciferase reporter vector (Figure $\mathbf{7 G}$ ). It was demonstrated that miR-874 mimics significantly decreased the relative luciferase activity of wild-type 3'UTR of STAT3, whereas no significant difference was observed for the mutated sequence (Figure $7 \mathbf{H}$ ). Therefore, our data demonstrated that inhibition of miR-874 suppressed the myocardial apoptosis in PPCM by targeting the STAT3/ JAK signaling pathway in Gaq transgenic mice.

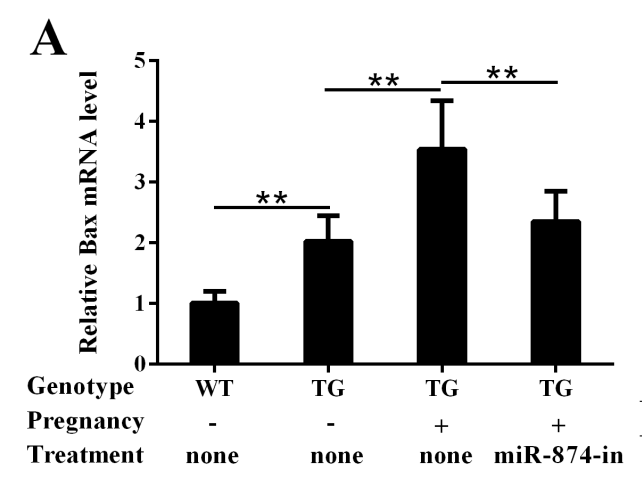

C
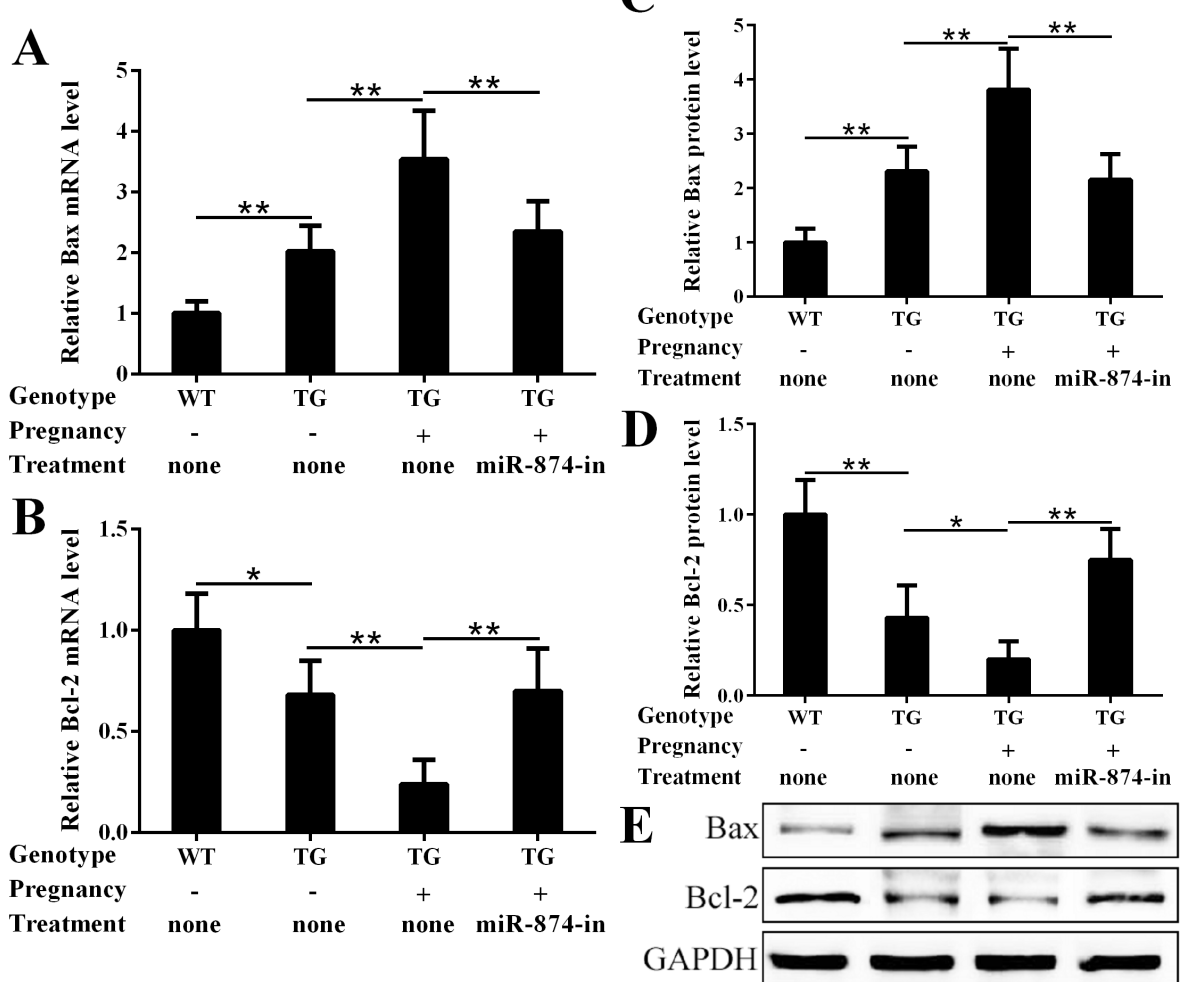

Figure 5. Inhibition of miR-874 ameliorated cardiomyocyte apoptosis in PPCM of Gaq transgenic mice. QRT-PCR was used to measure the mRNA levels of $\mathrm{Bax}(\mathrm{A})$ and $\mathrm{Bcl}-2(\mathrm{~B})$ from left ventricular myocardial tissues among different groups. Western blotting was used to measure the protein levels of $\mathrm{Bax}$ and $\mathrm{Bcl}-2$ from left ventricular myocardial tissues among different groups (C-E). Agematched, female non-pregnant wild-type (WT, $n=18)$ and Gaq transgenic mice $(G T, n=18)$, pregnant Gaq transgenic mice $(n=8)$ and Gaq transgenic mice treated with miR-874 antagonist (miR-874-in, $n=15$ ) were compared. Data are presented as mean \pm SD. ${ }^{*} p<0.05$, ${ }^{* *} p<$ 0.01 between the indicated groups. 
A

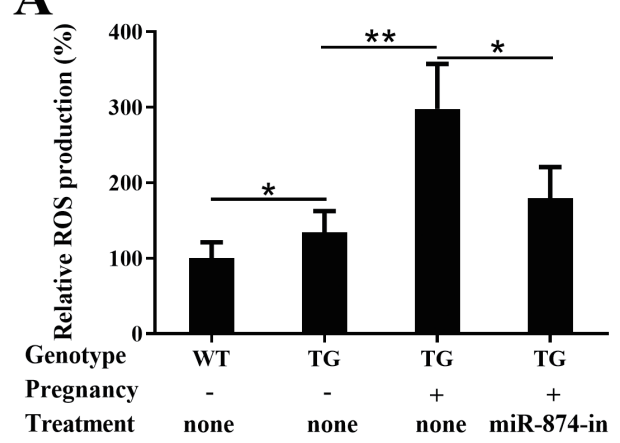

B

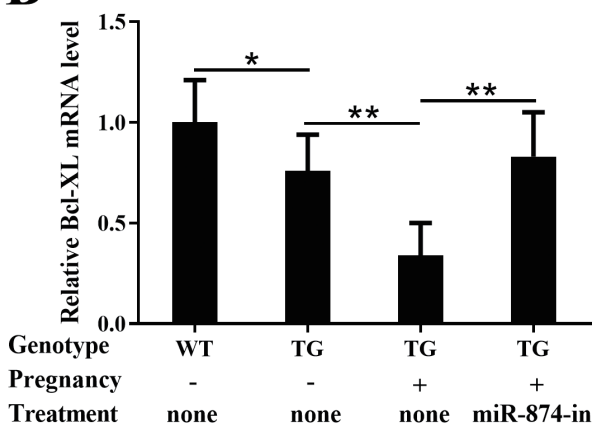

Figure 6. Measurement of ROS production in each group (A) and the relative Bcl-XL mRNA expressions in each group. Data are presented as mean $\pm S D$. ${ }^{*} p<0.05,{ }^{* *} p<0.01$ between the indicated groups.

\section{Discussion}

The generation and elimination of oxygen free radicals and active oxygen in the body are in a dynamic equilibrium state under normal circumstances, but in certain pathological situations, the generation of active oxygen beyond the body's ability to clear them will cause oxidative stress damage to tissues and cells (17). Many studies have shown that during the pathological process of heart diseases such as myocardial ischemia, cardiac remodeling and myocardial ischemia-reperfusion injury, more ROS and oxygen free radicals often accumulate. These accumulated ROS further cause cardiomyocyte necrosis and apoptosis, eventually aggravating the severity of the disease and forming a vicious cycle (18). Therefore, reducing the oxidative stress injury to cardiomyocytes and reducing apoptosis are of great clinical significance to improve the treatment effect of cardiovascular diseases and to improve cardiac functions.

MiRNAs participate in a variety of pathological and physiological processes such as apoptosis, proliferation and differentiation through the regulation of their target genes. Therefore, their roles in cardiovascular diseases have been valued by more and more researchers. MiRNAs are reported to participate in the pathogenesis of atherosclerotic plaques (21), vascular regeneration after myocardial infarction (22) and myocardial remodeling (23). Circulating miRNAs could also be used to diagnose cardiovascular diseases such as myocardial infarction $(24,25)$. MiR- 874 has been widely studied in tumors and various targets have been identified. Wang et al. reported that miR-874 could regulate myocardial necrosis via targeting caspase-8 (8). In addition, miR-874 inhibited epithelial-mesenchymal transition and metastasis in hepatocellular carcinoma by regulating sex-determining region Y-box 12, which regulated cell fate determination and embryonic development (26). Furthermore, miR874 alleviated inflammatory response and renal injury in diabetic nephropathy through targeting toll-like receptor-4 (27). Leong et al. reported that miR-874 expression was decreased in hepatocellular carcinoma and negatively regulated PIN1 expression, which modulated cell cycle progression, cell proliferation and apoptosis (28). These data suggest that miR-874 might regulate embryonic development and cell apoptosis in PPCM. Here, we reported that inhibition of miR-874 significantly improved cardiac functions and ameliorated cardiomyocyte apoptosis during PPCM in the Gaq transgenic mice. Inhibition of miR-874 significantly increased the survival and rescued the left ventricular dilation and contractile dysfunction in the pregnant Gaq transgenic mice. In addition, miR-874 inhibitor ameliorated cardiomyocyte apoptosis and regulated the expression of Bcl-2 and Bax.

STAT3 is an important signal transduction protein in cells, which is involved in a series of physiological activities such as apoptosis and proliferation of cells. When cells are stimulated, the SH2 domain of STAT3 binds to phosphorylated tyrosine residues on the receptor, and at the same time autophosphorylation occurs, enabling its entry into the nucleus and turning on its transcription activity (29). STAT3 is an important anti-apoptotic factor and STAT3 function in PPCM has been evaluated in various studies. The myocardial infarct area of STAT3 overexpressing myocardial tissue was significantly reduced after hypoxia-reperfusion injury (30). Overexpression of STAT3 in mouse cardiomyocytes could reduce apoptosis induced by hypoxia-reoxygenation injury (31). STAT3deficient mouse cardiomyocytes were more sensitive to oxidative stress injury and had a higher apoptosis rate (32). Angiotensin II AT1 receptor, which is Gq protein-coupled, is a major stimulus of JAK-STAT activation (33). Furthermore, GRK2 has been implicated in STAT3 regulation (34), as well as in cardiac anti-apoptosis/inflammation mediated by the $\beta 2$-adrenergic receptor (35). In the present study, we found that miR-874 inhibitor ameliorated cardiomyocyte apoptosis and regulated the expression of Bax and Bcl-2. Further, STAT3 was a direct target of miR874 , whose inhibition increased the expression and the activation of STAT3 in mouse cardiomyocytes. Our study hereby extends the knowledge on miR-874 in the context of PPCM by targeting STAT3. Other potential targets of miR-874 involved in the STAT3 signaling pathway, such as angiotensin II receptors and GRK2, need to be further investigated in future studies.

Overexpressed STAT3 could eliminate active oxygen in the body by upregulating the activity of the manganese superoxide enzyme, thereby reducing tissue damage 
A

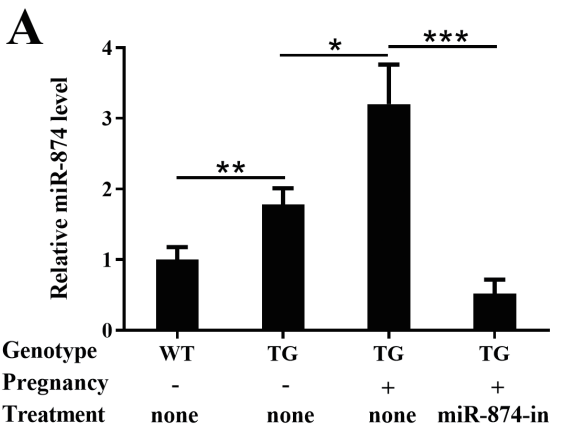

C

C

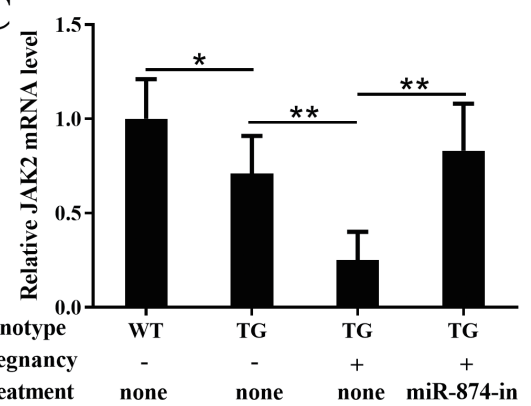

Treatment none none none miR-874-in

E

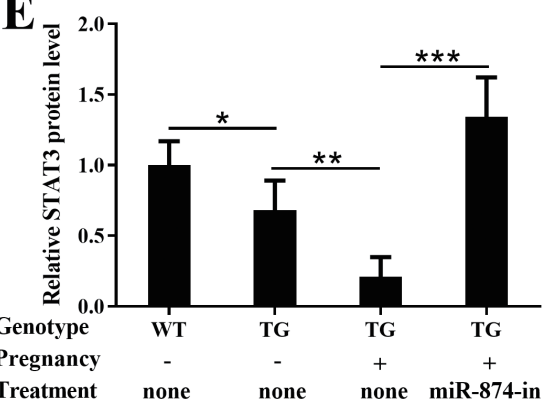

G

WT-Stat3 $55^{\prime}$...GAUAUCUGUAACCACAGGGCAA....3' mmu-miR-8743' ...AGCCAGGGAGCCCGGUCCCGUC...5'

Mut-Stat3 $5^{\prime}$...GAUAUCUGUAACCAGUCCCGUA....'
B

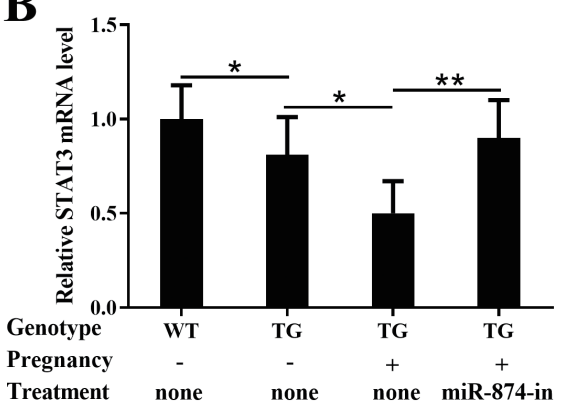

D

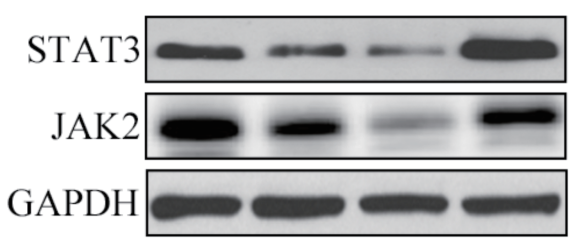

F

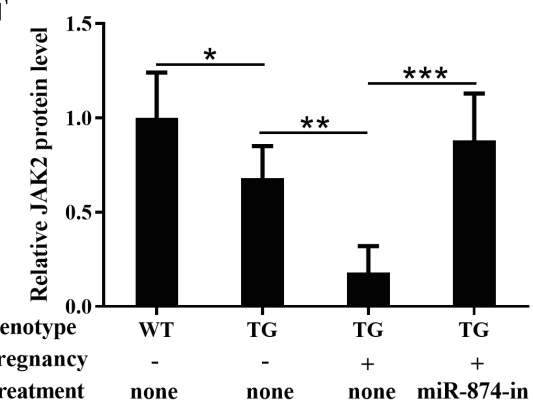

H

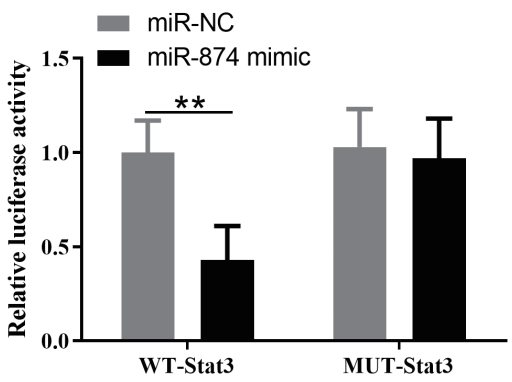

Figure 7. Inhibition of miR-874 targeted STAT3. QRT-PCR was used to measure the level of miR-874 (A), STAT3 (B) and JAK2 (C) among different groups. (D) Western blotting was used to measure the protein level of STAT3 and JAK2 among different groups and the relative expressions ( $E$ and F). (G) The suspected binding of miR-874 with the wild-type or mutant 3'-UTR region of STAT3 mRNA is shown. $(\mathrm{H})$ Luciferase activity after the transfection of the wild-type or mutant 3'-UTR region of STAT3 along with miR-874 mimics. Agematched, female non-pregnant wild-type $(W T, n=18)$ and Gaq transgenic mice $(G T, n=18)$, pregnant Gaq transgenic mice $(n=8)$ and Gaq transgenic mice treated with miR-874 antagonist (miR-874-in, $n=15$ ) were compared. Data are presented as mean \pm SD. ${ }^{*} p<0.05$, ${ }^{* *} p<$ 0.01 and ${ }^{* * *} p<0.001$ between the indicated groups.

caused by oxidative stress (31). Prolactin is one of the important hormones to maintain normal physiological activities among pregnant women. Oxidative stress could cleavage full length prolactin into $16 \mathrm{k}$ Da prolactin fragments by stimulating the protein expression of cathepsin D in cardiomyocytes, while 16k Da prolactin fragments in the body could promote the apoptosis of vascular endothelial cells and prevent angiogenesis by upregulating miR-146a $(36,37)$. The treatment using kiosk (which could inhibit the release of prolactin) returned the plasma miR-146a levels of PPCM patients to normal levels $(13,37)$. In addition, animal experiments have confirmed that mice with reduced anti-oxidative stress capacity after special treatment show symptoms similar to dilated cardiomyopathy after delivery. In this study, we found that the ROS production was increased in Gaq transgenic mice compared with wild-type mice before pregnancy, and the pregnant Gaq transgenic mice showed further increased ROS production, which was consistent with previous studies. Notably, miR-874 
inhibitor treatment significantly decreased the ROS production to a level similar to the non-pregnant Gaq transgenic mice. Our research suggests that miR-874 inhibitor could be utilized for the clinical treatment of PPCM, bringing new hope for the clinical management of PPCM.

\section{Conclusion}

In conclusion, our results have demonstrated that miR874 inhibition significantly increases the survival and cardiac functions of pregnant Gaq transgenic mice. In addition, miR-874 inhibitor ameliorates cardiomyocyte apoptosis and regulates the expression of Bax and Bcl-2. Further, STAT3 is a direct target of miR-874 and miR-874 antagonist increases the expression and the activation of STAT3. Our findings reveal the potential biomarkers for cardiovascular disease and novel therapeutic targets for the treatment of PPCM.

\section{Conflict of interest}

The authors declare that they have no conflicts of interest to disclose.

\section{Funding}

The work was supported by the National Natural Science Foundation of China (N0.81873816, NO. 82071629): Natural Science Foundation of Shanghai Municipal Health Commission(NO. 202040128); Natural science foundation of Pudong Municipal Health Commission of Shanghai (NO. PW2019D-13).

\section{Supplementary Information}

The supplemental material can be downloaded online at: https://stemedicine.org/index.php/stem/article/view/75

\section{References}

1 Honigberg MC, Givertz MM. Peripartum cardiomyopathy. BMJ. 2019;364:k5287.

2 Cunningham FG, Byrne JJ, Nelson DB. Peripartum cardiomyopathy. Obstet Gynecol. 2019;133:167-179.

3 Koenig T, Hilfiker-Kleiner D, Bauersachs J. Peripartum cardiomyopathy. Herz. 2018;43:431-437.

4 Hilfiker-Kleiner D, Sliwa K. Pathophysiology and epidemiology of peripartum cardiomyopathy. Nat Rev Cardiol. 2014;11:364-370.

5 Goland S, Elkayam U. Peripartum cardiomyopathy: approach to management. Curr Opin Cardiol. 2018;33:347-353.

6 Hayes J, Peruzzi PP, Lawler S. MicroRNAs in cancer: biomarkers, functions and therapy. Trends Mol Med. 2014;20:460-469

7 Liu X, You L, Zhou R, Zhang J. Significant association between functional microRNA polymorphisms and coronary heart disease susceptibility: a comprehensive meta-analysis involving 16484 subjects. Oncotarget. 2017;8:5692-5702.

8 Wang K, Liu F, Zhou LY, Ding SL, Long B, Liu CY, et al. miR874 regulates myocardial necrosis by targeting caspase-8. Cell Death Dis. 2013;4:e709.

9 Chen PJ, Shang AQ, Yang JP, Wang WW. microRNA-874 inhibition targeting STAT3 protects the heart from ischemiareperfusion injury by attenuating cardiomyocyte apoptosis in a mouse model. J Cell Physiol. 2019;234:6182-6193.

10 Zhang TJ, Guo RX, Li X, Wang YW, Li YJ. Tetrandrine cardioprotection in ischemia-reperfusion (I/R) injury via JAK3/ STAT3/Hexokinase II. Eur J Pharmacol. 2017;813:153-160.
11 Stapel B, Kohlhaas M, Ricke-Hoch M, Haghikia A, Erschow S, Knuuti J, et al. Low STAT3 expression sensitizes to toxic effects of beta-adrenergic receptor stimulation in peripartum cardiomyopathy. Eur Heart J. 2017;38:349-361.

12 Ricke-Hoch M, Bultmann I, Stapel B, Condorelli G, Rinas $\mathrm{U}$, Sliwa K, et al. Opposing roles of Akt and STAT3 in the protection of the maternal heart from peripartum stress. Cardiovasc Res. 2014;101:587-596.

13 Hilfiker-Kleiner D, Kaminski K, Podewski E, Bonda T, Schaefer A, Sliwa K, et al. A cathepsin D-cleaved 16 kDa form of prolactin mediates postpartum cardiomyopathy. Cell. 2007;128:589-600.

14 Sanchez-Fernandez G, Cabezudo S, Garcia-Hoz C, Beninca C, Aragay AM, Mayor F, Jr., et al. Galphaq signalling: the new and the old. Cell Signal. 2014;26:833-848.

15 Hayakawa Y, Chandra M, Miao W, Shirani J, Brown JH, Dorn GW, 2nd, et al. Inhibition of cardiac myocyte apoptosis improves cardiac function and abolishes mortality in the peripartum cardiomyopathy of Galpha(q) transgenic mice. Circulation. 2003;108:3036-3041.

16 D'Angelo DD, Sakata Y, Lorenz JN, Boivin GP, Walsh RA, Liggett SB, et al. Transgenic Galphaq overexpression induces cardiac contractile failure in mice. Proc Natl Acad Sci U S A. 1997;94:8121-8126.

17 Popolo A, Autore G, Pinto A, Marzocco S. Oxidative stress in patients with cardiovascular disease and chronic renal failure. Free Radic Res. 2013;47:346-356.

18 Elahi MM, Kong YX, Matata BM. Oxidative stress as a mediator of cardiovascular disease. Oxid Med Cell Longev. 2009;2:259-269.

19 Wightman B, Ha I, Ruvkun G. Posttranscriptional regulation of the heterochronic gene lin-14 by lin-4 mediates temporal pattern formation in C. elegans. Cell. 1993;75:855-862.

20 Xia W, Cao G, Shao N. Progress in miRNA target prediction and identification. Sci China C Life Sci. 2009;52:1123-1130.

21 Siomi H, Siomi MC. Posttranscriptional regulation of microRNA biogenesis in animals. Mol Cell. 2010;38:323-332.

22 Fabian MR, Sonenberg N, Filipowicz W. Regulation of mRNA translation and stability by microRNAs. Annu Rev Biochem. 2010;79:351-379.

23 Jayaswal V, Lutherborrow M, Yang YH. Measures of association for identifying microRNA-mRNA pairs of biological interest. PLoS One. 2012;7:e29612.

24 Jones CI, Zabolotskaya MV, King AJ, Stewart HJ, Horne GA, Chevassut TJ, et al. Identification of circulating microRNAs as diagnostic biomarkers for use in multiple myeloma. $\mathrm{Br} \mathrm{J}$ Cancer. 2012;107:1987-1996.

25 Anand S. A brief primer on microRNAs and their roles in angiogenesis. Vasc Cell. 2013;5:2.

26 Jiang T, Guan LY, Ye YS, Liu HY, Li R. MiR-874 inhibits metastasis and epithelial-mesenchymal transition in hepatocellular carcinoma by targeting SOX12. Am J Cancer Res. 2017;7:1310-1321.

27 Yao T, Zha D, Gao P, Shui H, Wu X. MiR-874 alleviates renal injury and inflammatory response in diabetic nephropathy through targeting toll-like receptor-4. J Cell Physiol. 2018;234:871-879.

28 Leong KW, Cheng CW, Wong CM, Ng IO, Kwong YL, Tse E. miR-874-3p is down-regulated in hepatocellular carcinoma and negatively regulates PIN1 expression. Oncotarget. 2017;8:11343-11355.

29 Hillmer EJ, Zhang H, Li HS, Watowich SS. STAT3 signaling in immunity. Cytokine Growth Factor Rev. 2016;31:1-15.

30 Oshima Y, Fujio Y, Nakanishi T, Itoh N, Yamamoto Y, Negoro $\mathrm{S}$, et al. STAT3 mediates cardioprotection against ischemia/ reperfusion injury through metallothionein induction in the heart. Cardiovasc Res. 2005;65:428-435.

31 Negoro S, Kunisada K, Fujio Y, Funamoto M, Darville MI, Eizirik DL, et al. Activation of signal transducer and activator of transcription 3 protects cardiomyocytes from hypoxia/reoxygenation-induced oxidative stress through the 
upregulation of manganese superoxide dismutase. Circulation. 2001;104:979-981.

32 Barry SP, Townsend PA, McCormick J, Knight RA, Scarabelli TM, Latchman DS, et al. STAT3 deletion sensitizes cells to oxidative stress. Biochem Biophys Res Commun. 2009;385:324-329.

33 Capote LA, Mendez Perez R, Lymperopoulos A. GPCR signaling and cardiac function. Eur J Pharmacol. 2015;763:143-148.

34 Palikhe S, Ohashi W, Sakamoto T, Hattori K, Kawakami M, Andoh T, et al. Regulatory Role of GRK2 in the TLR Signaling-Mediated iNOS Induction Pathway in Microglial Cells. Front Pharmacol. 2019;10:59.
35 Salazar NC, Vallejos X, Siryk A, Rengo G, Cannavo A, Liccardo D, et al. GRK2 blockade with betaARKct is essential for cardiac beta2-adrenergic receptor signaling towards increased contractility. Cell Commun Signal. 2013;11:64.

36 Roberg K, Ollinger K. Oxidative stress causes relocation of the lysosomal enzyme cathepsin D with ensuing apoptosis in neonatal rat cardiomyocytes. Am J Pathol. 1998;152:1151-1156.

37 Halkein J, Tabruyn SP, Ricke-Hoch M, Haghikia A, Nguyen $N Q$, Scherr M, et al. MicroRNA-146a is a therapeutic target and biomarker for peripartum cardiomyopathy. J Clin Invest. 2013;123:2143-2154. 